\title{
Colonial morphology of Escherichia coli: impact of detection in clinical specimens
}

\author{
Lucia Barcella, Angelo Pasquale Barbaro, Santa Beatrice Rogolino \\ Department of Microbiology, Bianchi Melacrino Morelli Hospital, Reggio Calabria, Italy
}

\begin{abstract}
Summary
As part of bacteriological identification, the macroscopic observation of the colonies on a culture medium is a critical step as it allows the clinical microbiologist to conduct a primary screening of microorganisms and in some cases even to identify them. This study shows how the macroscopic observation of the colonies, in particular of the colonies of Escherichia coli isolated on MacConkey agar from various clinical specimens, allowed to identify 5 classes of morphological variants of the colonies or colonial morphology, thanks to the knowledge and the experience gained by the clinical microbiologist.
\end{abstract}

\section{Introduction}

As part of bacteriological identification, the macroscopic observation of the colonies on a culture medium is a critical step, as it allows the clinical microbiologist to conduct a primary screening of the microorganisms.

It provides for a two-stage approach: i) a presumptive or preliminary

Correspondence: Lucia Barcella, Department of Microbiology, Bianchi Melacrino Morelli Hospital, via Sbarre Inferiori 417, 89100 Reggio Calabria, Italy.

Tel.: +39.340 .9418543$

E-mail: luciabarcella@gmail.com

Key words: Escherichia coli; colonial morphology; macroscopic expression; MacConkey agar.

Contributions: the authors contributed equally.

Conflict of interest: the authors declare no potential conflict of interest.

Received for publication: 17 November 2015.

Revision received: 1 February 2016.

Accepted for publication: 1 February 2016.

(C) Copyright L. Barcella et al., 2016

Licensee PAGEPress, Italy

Microbiologia Medica 2016; 31:5636

doi:10.4081/mm.2016.5636

This article is distributed under the terms of the Creative Commons Attribution Noncommercial License (by-nc 4.0) which permits any noncommercial use, distribution, and reproduction in any medium, provided the original author(s) and source are credited. identification, based on macroscopic and microscopic observation of the colonies; ii) the confirmation of the results of the presumptive identification through secondary tests (biochemical, serological, molecular tests), such as those provided by commercial identification systems (definitive identification).

This study shows how the macroscopic observation of the colonies, in particular those of Escherichia coli grown on MacConkey agar from various clinical specimens, allowed to identify five classes of morphological colony variants or colonial morphology (CM), thanks to the knowledge and the experience gained by the clinical microbiologist (also Rhodes K. S., in 1966, defined them this way) (4).

\section{Materials and Methods}

The study, aiming to evaluate the incidence and the antibiotic-resistance profiles of the CM of $885 \mathrm{E}$. coli strains collected from different clinical samples of 712 patients, was carried out between October 2014 and May 2015.

A total of 885 E. coli strains was studied divided as follows: i) 645 were from inpatients, obtained from faecal material $(n=285)$, urine $(n=270)$, respiratory specimens $(n=20)$, effusions $(n=16)$, venous catheters $(n=13)$, blood cultures $(n=13)$, swabs and other samples $(n=28) ; i i)$ 240 were from outpatients, obtained from urine $(n=128)$, faeces $(\mathrm{n}=97)$, swabs and other samples $(\mathrm{n}=10)$, respiratory specimens $(\mathrm{n}=5)$.

The E. coli strains, at first were isolated in pure culture on MacConkey Agar and then, after 24 hours of incubation at $37^{\circ} \mathrm{C}$, subjected to identification and antibiogram analysis by the semi-automated System Vitek2 (bioMérieux, Marcy l’Etoile, France).

\section{Results and Discussion}

Between October 2014 and June 2015, 885 strains of $E$. coli have been studied, 645 (73\%) being from hospitalized patients and 240 (27\%) from outpatients.

The results of this work have been achieved from the observation of the macroscopic colonies of $E$. coli on MacConkey agar after no more than 24 hours of incubation at $37^{\circ} \mathrm{C}$ in fact, as already Hasman (2) in 1999 noted, over this time the observed CM could be converted into one another.

Most of the collected $E$. coli isolates (n=835, 94\%) showed bright pink colonies on MacConkey agar, while 50 strains (6\%) produced yellow-amber colonies (Figure 1).

The macroscopic observation of $E$. coli strains grown on agar MacConckey allowed us to identify five main types of CM. Depending on the macroscopic aspect of the colonies, these CM series have been divided into five classes, as follows: CM I or domed (representing the 
typical morphology of $E$. coli colonies); CM II or umbilicated; CM III or flared; CM IV or cercinate; CM V or not fermenting lactose.

The percentages identified for each single morphology are reported in Table 1.

The class CM I represents $28 \%$ of the isolated $E$. coli strains ( $\mathrm{n}=250)$ and it shows a dome-shaped appearance, firm, raised and opaque (Figure $2 \mathrm{a}$ ). The class CM II represents $32 \%$ of the isolated strains $(\mathrm{n}=283)$ and shows a similar dome shaped but in the middle presents a slight depression and inside this, middle located, has a little bump point (Figure $2 \mathrm{~b}$ ). The class CM III represents $27 \%$ of isolated strains $(\mathrm{n}=243)$, appears flat and flared, often with uneven edges, with reduced thickness and larger than the classes CM I and CM II (Figure 2c). The class CM IV, representing 7\% $(\mathrm{n}=59)$ isolated, is similar to the class CM II but shows significantly raised edges, a deeper central depression and a more pronounced central core (Figure $2 \mathrm{~d}$ ). These four classes appear bright pink colour on MacConkey agar, suggestive of bacterium ability to use the lactose present in the culture medium.

The class CM V is similar to the CM III class in dimensions and morphological characteristics but appears yellow-amber on MacConkey agar (Figure 3), a clear sign of the inability to use lactose; this class is the rarest, as it is identified only in $6 \%$ of the isolates $(n=50)$.

The CM II could be defined as the dominant CM, because it's the more frequently identified among the $E$. coli strains evaluated in this study; the CM I and the CM III also represent a large percentage of the isolates, but they appeared in a lower proportion than the CM II. The CM IV and V CM are shown only in a smaller group of isolates.

It is interesting to note that a relevant proportion of the clinical $E$. coli isolates shown the coexistence of two or more different classes of
E. coli $\mathrm{CM}$ : of 382 examined faecal material samples, 54 (14\%) showed different class of CM. In $82 \%$ of these cases the CM III class has been the most frequently identified.

No significant differences in CM classes were identified among E.coli isolates obtained from in- and outpatients as reported in Table 2 below.

The biochemical profiles of the $885 \mathrm{E}$. coli strains isolated, regardless of the class of CM, was obtained by Vitek2 System through the use of the GN Card (Gram Negative Card, bioMérieux). The percentages of positivity to the various biochemical tests relating to the five different classes of $E$. coli $\mathrm{CM}$ are reported in Table 3 .

The five classes of CM showed coherent biochemical profiles (Table 3 ). The only relevant finding was the $100 \%$ positivity identified in $100 \%$ of the CM V isolates for the so called ELLM biochemical test, which, using Ellman's reagent (DTNB or 5,5'-dithio-bis [2- nitroben-

Table 1. Percentage of isolation for the five $E$. coli colonial morphology (CM) classes.

\begin{tabular}{lcc}
\hline E. coli CM & N. samples & \% isolation \\
I & 250 & 28 \\
II & 283 & 32 \\
\hline III & 243 & 27 \\
IV & 59 & 7 \\
\hline V & 50 & 6 \\
Total & 885 & 100 \\
\hline
\end{tabular}
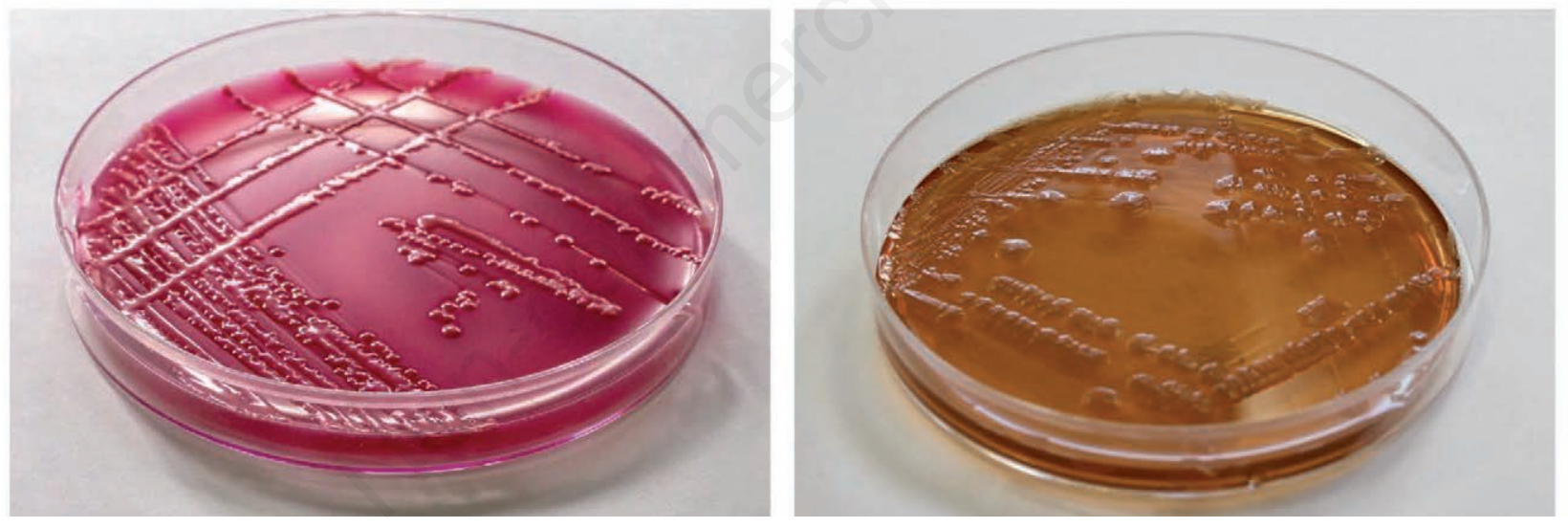

Figure 1. E. coli pink colonies and yellow-amber colonies.
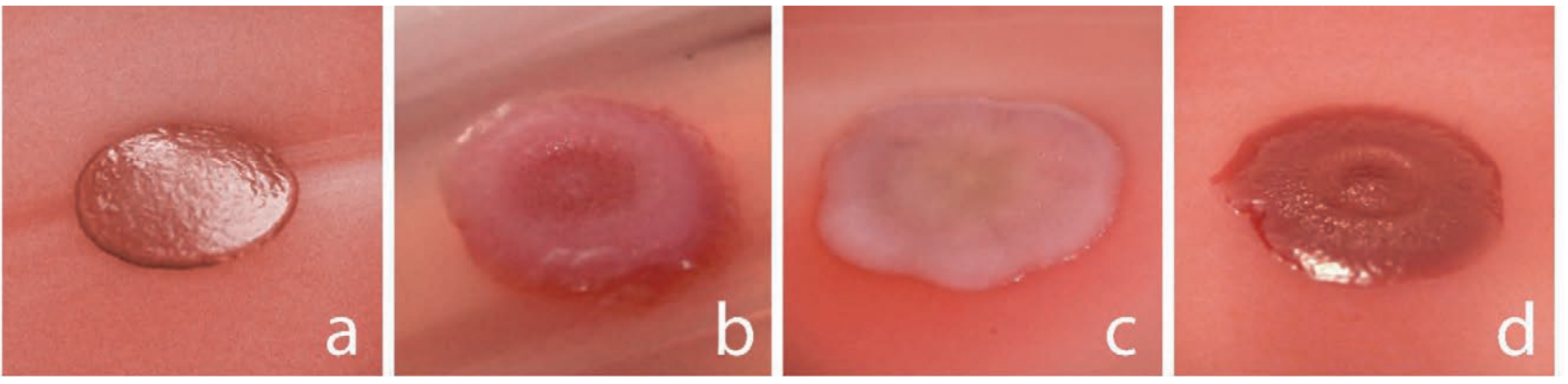

Figure 2. E. coli colonial morphology (CM): CM I (a), CM II (b), CM III (c) and CM IV (d) classes. 
zoic acid]), quantifies the thiols that are molecules derived from the metabolism of methionine. The remaining CM classes showed a much lower percentage of strains positive to the ELLM test, ranging from 1.1 (CM II) to 5.2\% (CM IV).

Data shown in Table 3 also indicate that $20 \%$ of the strains in the class CM V $(n=10)$ are lacking Beta-Galactosidase given their negativity to the BGAL test.

The antibiotic susceptibility profiles of the 885 E. coli strains studied, were obtained by using the automated System Vitek2 with the Cards type 202/204 (bioMérieux); the results (in term of \% sensitivity to each individual antimicrobial evaluated) are summarized in Figure 4.

Table 4 reports the detail about \% sensitivity of the $E$. coli isolated divided by their CM class.

Overall, there were no significant differences of antibiotic susceptibility tested between the five classes of CM. Most of the isolates, as expected and according to the data from literature, are resistant to ciprofloxacin and ampicillin. $29.5 \%$ of the studied bacteria were also resistant to trimethoprim/sulfamethoxazole, while the resistance to -

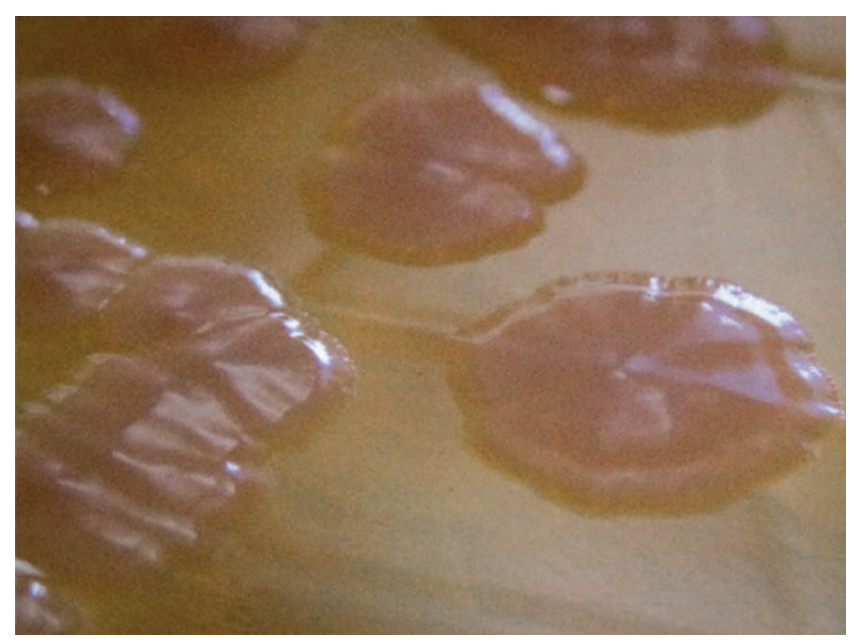

Figure 3. E. coli colonial morphology V class.

Table 2. Percentage of isolation of the five $E$. coli colonial morphology (CM) classes from inpatients and outpatients.

\begin{tabular}{|c|c|c|c|c|c|c|c|c|c|c|c|}
\hline \multirow{2}{*}{ Population } & \multicolumn{2}{|c|}{ CM I } & \multicolumn{2}{|c|}{ CM II } & \multicolumn{2}{|c|}{ CM III } & \multicolumn{2}{|c|}{ CM IV } & \multicolumn{2}{|c|}{ CM V } & \multirow[t]{2}{*}{ Total } \\
\hline & N. & $\%$ & N. & $\%$ & N. & $\%$ & N. & $\%$ & N. & $\%$ & \\
\hline Hospitalised & 200 & 31 & 208 & 32.3 & 168 & 26 & 39 & 6 & 30 & 4.7 & 645 \\
\hline Outpatients & 50 & 21.1 & 75 & 31.3 & 75 & 31.3 & 20 & 8.3 & 20 & 8.3 & 240 \\
\hline
\end{tabular}

Table 3. Percentage of positivity to biochemical tests of the five E. coli colonial morphology (CM) classes.

\begin{tabular}{|c|c|c|c|c|c|c|c|c|c|c|c|}
\hline $\begin{array}{l}\text { Biochemical } \\
\text { tests }\end{array}$ & CM I & CM II & CM III & CM IV & CM V & $\begin{array}{l}\text { Biochemical } \\
\text { tests }\end{array}$ & CM I & CM II & CM III & CM IV & CM V \\
\hline APPA & 0 & 0 & 0 & 0 & 0 & IARI & 0 & 0 & 0 & 0 & 0 \\
\hline $\mathrm{H} 2 \mathrm{~S}$ & 0.9 & 0 & 1.3 & 0 & 0 & dGLU & 99.1 & 100 & 99.3 & 94.7 & 98 \\
\hline BGLU & 0 & 0 & 0 & 0 & 0 & dMNE & 99.1 & 100 & 99.3 & 94.7 & 100 \\
\hline ProA & 30 & 17.2 & 27.3 & 31.6 & 14 & TyrA & 83.6 & 77.4 & 90 & 68.4 & 68 \\
\hline SAC & 56.4 & 68.9 & 55.3 & 79 & 50 & CIT & 0.9 & 0 & 0 & 0.7 & 0 \\
\hline ILATk & 49.1 & 23.6 & 40.6 & 31.6 & 32 & NAGA & 0 & 0 & 0 & 0 & 0 \\
\hline GlyA & 0.9 & 0 & 4 & 5.2 & 2 & IHISa & 0 & 0 & 0 & 0 & 0 \\
\hline 0129R & 76.4 & 72 & 72.6 & 52.6 & 68 & ELLM & 2.7 & 1.1 & 4 & 5.2 & 100 \\
\hline ADO & 6.4 & 2.1 & 5.3 & 0 & 0.8 & dCEL & 0 & 0 & 0 & 0 & 0 \\
\hline BNAG & 0 & 0 & 0 & 0 & 0 & GGT & 3.6 & 0 & 3.3 & 0 & 2 \\
\hline dMAL & 96.4 & 96.8 & 98 & 100 & 98 & BXYL & 0 & 0 & 0 & 0 & 0 \\
\hline LIP & 0 & 0 & 0 & 0 & 0 & URE & 2.7 & 0 & 1.3 & 10.5 & 2 \\
\hline dTAG & 29.1 & 32.3 & 21.3 & 73.7 & 16 & MNT & 0 & 0 & 0 & 0 & 0 \\
\hline AGLU & 0 & 0 & 0 & 0 & 0 & AGAL & 80.9 & 86 & 89.3 & 78.9 & 84 \\
\hline ODC & 62.7 & 78.5 & 50 & 89.5 & 70 & CMT & 98.2 & 97.8 & 98.6 & 100 & 94 \\
\hline GGAA & 0 & 0 & 0 & 0 & 0 & ILATa & 1.8 & 1.1 & 2.7 & 0 & 0 \\
\hline PyrA & 0 & 0 & 0 & 0 & 0 & BGAL & 100 & 100 & 100 & 100 & 80 \\
\hline AGLTp & 0 & 0 & 0 & 0 & 0 & OFF & 95.4 & 98.9 & 98 & 94.7 & 84 \\
\hline dMAN & 99.1 & 98.9 & 99.3 & 94.7 & 94 & BAIap & 0 & 0 & 0 & 0 & 0 \\
\hline PLE & 0 & 0 & 0.7 & 0 & 0 & dSOR & 97.3 & 95.7 & 97.3 & 100 & 96 \\
\hline dTRE & 99.1 & 98.9 & 100 & 94.7 & 96 & $5 \mathrm{KG}$ & 22.7 & 38.7 & 35.4 & 31.6 & 24 \\
\hline SUCT & 93.6 & 82.8 & 88 & 84.21 & 54 & PHOS & 1.8 & 1.1 & 6.7 & 5.3 & 6 \\
\hline LDC & 91.8 & 94.6 & 87.3 & 89.5 & 96 & BGUR & 84.5 & 89.2 & 85.3 & 84.2 & 84 \\
\hline IMLTa & 3.6 & 1 & 2.7 & 0 & 0 & & & & & & \\
\hline
\end{tabular}


lactamines with Beta-Lactamases inhibitors ranged between 8 and $20 \%$. Furthermore gentamicin was the less effective among the aminoglycosides. Only $17.6 \%$ of $E$. coli strains (n=156) were ES L (ExtendedSpectrum Beta-lactamases) producer.

\section{Conclusions}

The possibility to distinguish different $E$. coli $\mathrm{CM}$, was already described by Rhodes in 1966 (4) on Tergitol-7 Agar and this has been confirmed in this study, thus highlighting the large versatility of macroscopic expression of these bacteria (7).
Considering that CM II type represents the most frequently identified CM among the $E$. coli strains studied, we could probably speculate that the classes less frequent of CMs likely differentiated more recently than CM II, and this could justify their low rate of identification. Based also on data by Drucker and Whittaker (1), we can assume that the different classes of $\mathrm{CM}$ are not dependent on the metabolic profiles, that apparently differ only in the class CM V (characterized by the inability to ferment the lactose and by a stronger metabolism of methionine), but this could rather depend on the different arrangement that individual bacterial cells implement during the process of colony development (6).

In our opinion, the characterization of the features of different bacterial isolates should not be based only on the study of biochemical profiles and antibiotic susceptibility. Standard procedures should be sup-

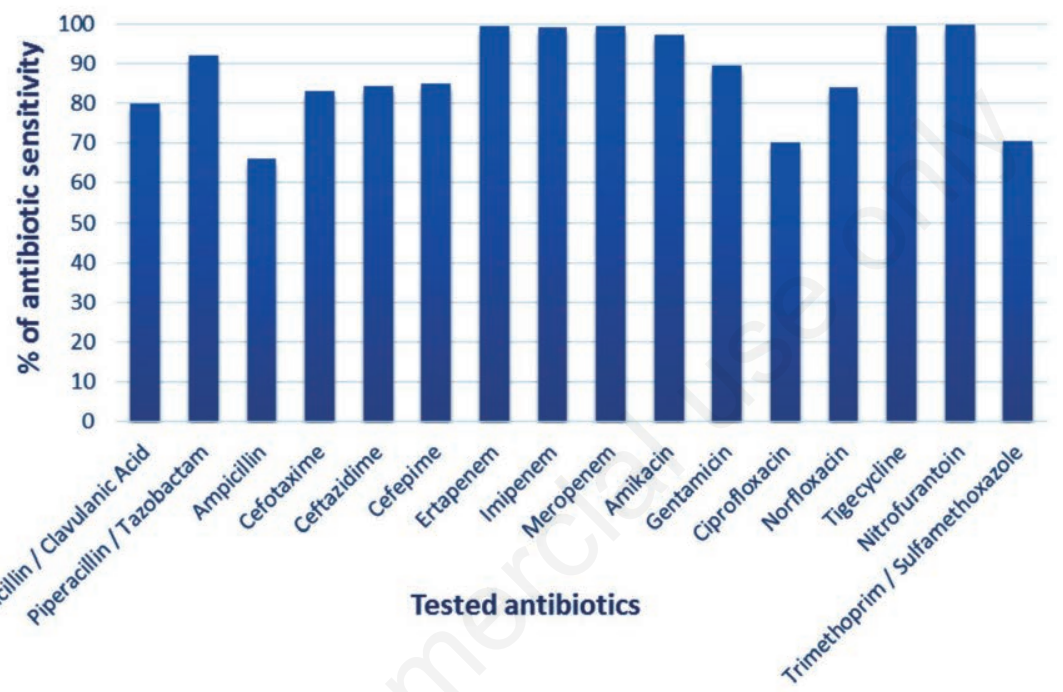

Figure 4. Percentage of the total antibiotic susceptibility of $E$. coli isolated strains.

Table 4. Percentages of antibiotic sensitivity of the five $E$. coli colonial morphology (CM) classes.

\begin{tabular}{|c|c|c|c|c|c|c|}
\hline \multirow{2}{*}{ Antibiotics } & \multirow[b]{2}{*}{ Tot $E$. coli } & \multicolumn{4}{|c|}{$\%$ of sensitivity against tested antibiotics } & \multirow[b]{2}{*}{ CM V } \\
\hline & & CM I & CM II & CM III & CM IV & \\
\hline Amoxicillin/Clavulanic Acid & 80 & 74 & 87.3 & 79.8 & 76.3 & 82 \\
\hline Piperacillin/Tazobactam & 92 & 89.2 & 94.3 & 92.2 & 91.5 & 92 \\
\hline Ampicillin & 66.2 & 62 & 74.6 & 63.8 & 61 & 58 \\
\hline Cefotaxime & 83 & 75.6 & 90.1 & 85.2 & 72.9 & 82 \\
\hline Ceftazidime & 84.5 & 77.6 & 89.7 & 87.2 & 76.3 & 86 \\
\hline Cefepime & 85.1 & 79.2 & 90.1 & 87.2 & 78 & 84 \\
\hline Ertapenem & 99.4 & 99.2 & 100 & 98.8 & 100 & 100 \\
\hline Imipenem & 99.2 & 98.8 & 100 & 98.3 & 100 & 100 \\
\hline Meropenem & 99.5 & 99.6 & 99.3 & 99.6 & 100 & 100 \\
\hline Amikacin & 97.3 & 95.6 & 98.6 & 96.7 & 98.3 & 100 \\
\hline Gentamicin & 89.6 & 86.8 & 92.2 & 91.8 & 88.13 & 80 \\
\hline Ciprofloxacin & 70.3 & 60.4 & 82.3 & 67.5 & 64.4 & 72 \\
\hline Norfloxacin & 84.1 & 84 & 89.7 & 81.1 & 83 & 68 \\
\hline Tigecycline & 99.3 & 99.2 & 100 & 98.8 & 100 & 98 \\
\hline Nitrofurantoin & 99.7 & 99.6 & 100 & 99.6 & 100 & 98 \\
\hline Trimethoprim/Sulfamethoxazole & e 70.5 & 63.2 & 74.6 & 72.8 & 74.6 & 68 \\
\hline
\end{tabular}


ported and complemented by the observation of the morphology and colonies disposition. This old new way to discriminate different E.coli strains could be the starting point for a deeper investigation the link between the macroscopic expression and microscopic features of bacteria (5). This work could thus be an innovative stimulus for Clinical Microbiologists to better observe and study the microorganisms not only considering their possible (pathogenic) effects on the human host, but also as a guide to understand a world still largely unknown.

\section{References}

1. Drucker DB, Whittaker DK. Microstructure of colonies of rodshaped bacteria. J Bacteriol 1971;108:515-25.
2. Hasman H, Schembri MA, Klemm P. Antigen 43 and type 1 fimbriae determine colony morphology of Escherichia coli K-12. J Bacteriol 2000;182:1089-95.

3. Murray P, Tenover FC, Pfaller MA, eds. Manual of clinical microbiology. 6th Edition. Washington: ASM Press; 2009.

4. Rhodes KS. Colonial morphology of Escherichia coli on tergitol-7 medium. Appl Microbiol 1966;14:152-5.

5. Shapiro JA. Organization of developing Escherichia coli colonies viewed by scanning electron microscopy. J Bacteriol 1987;169:142-56.

6. Su PT, Liao CT, Roan JR, et al. Bacterial colony from two-dimensional division to three-dimensional development. PLoS One 2012; 7:e48098

7. Zinnah MA, Bari MR, Islam MT, et al. Characterization of Escherichia coli isolated from samples of different biological and environmental sources. Bangladesh J Vet Med 2007;5:25-32. 